\title{
Structural Diversity Among Fungal Hyphae: Insights into Cell Growth and phylogeny
}

David Lowry, Bonnie Saucedo, Dan MacLean, Terrence A. Oneil, Jeff Propster, Brant Unger, and Robert W. Roberson

School of Life Sciences, Arizona State University, Tempe, AZ USA 85287-4501

The defining feature of filamentous fungi is the hypha; a tube-shaped cell that grows at its tip (Fig. 1). The mode of hyphal growth is a complex process that has allowed the fungi to successfully utilize a wide range of ecological habitats and develop multiple lifestyles. The hyphal cytoplasm contains many of the organelles and sub-cellular inclusions found in other heterotrophic eukaryotic organisms (Figs 2, 3). However, because of their mode of growth and diverse interactions with the ecosystem, hyphae also maintain a cytoplasmic structure and contain elements that are unique to the eukaryotes. Some cytoplasmic features show significant structural plasticity within the fungi themselves or are present in only certain fungal groups. These elements (e.g., Spitzenkörper, Figs. 24) are of particular importance in understanding aspects of hyphal growth and are being used as indicators of fungal evolution [1].

In this presentation, light and electron microscopy are used to review cytoplasmic features of hyphae from diverse taxonomic groups. Characteristics of cytoplasmic order, behavior, and selected organelles that are relevant to understanding hyphal growth and fungal phylogeny will be presented.

\section{References}

[1] Roberson, R.W., Abril, M., Blackwell, M., Letcher, P., McLaughlin, D.J., Mouriño-Pérez, R.R., Riquelme, M., Uchida, M. 2009. Hyphal Structure, Chapter 2. In: Cellular and Molecular Biology of Filamentous Fungi. Eds. Borkovich, K. and Ebbole, D. ASM Press, Washington, D.C. (pp. 8 - 27). 


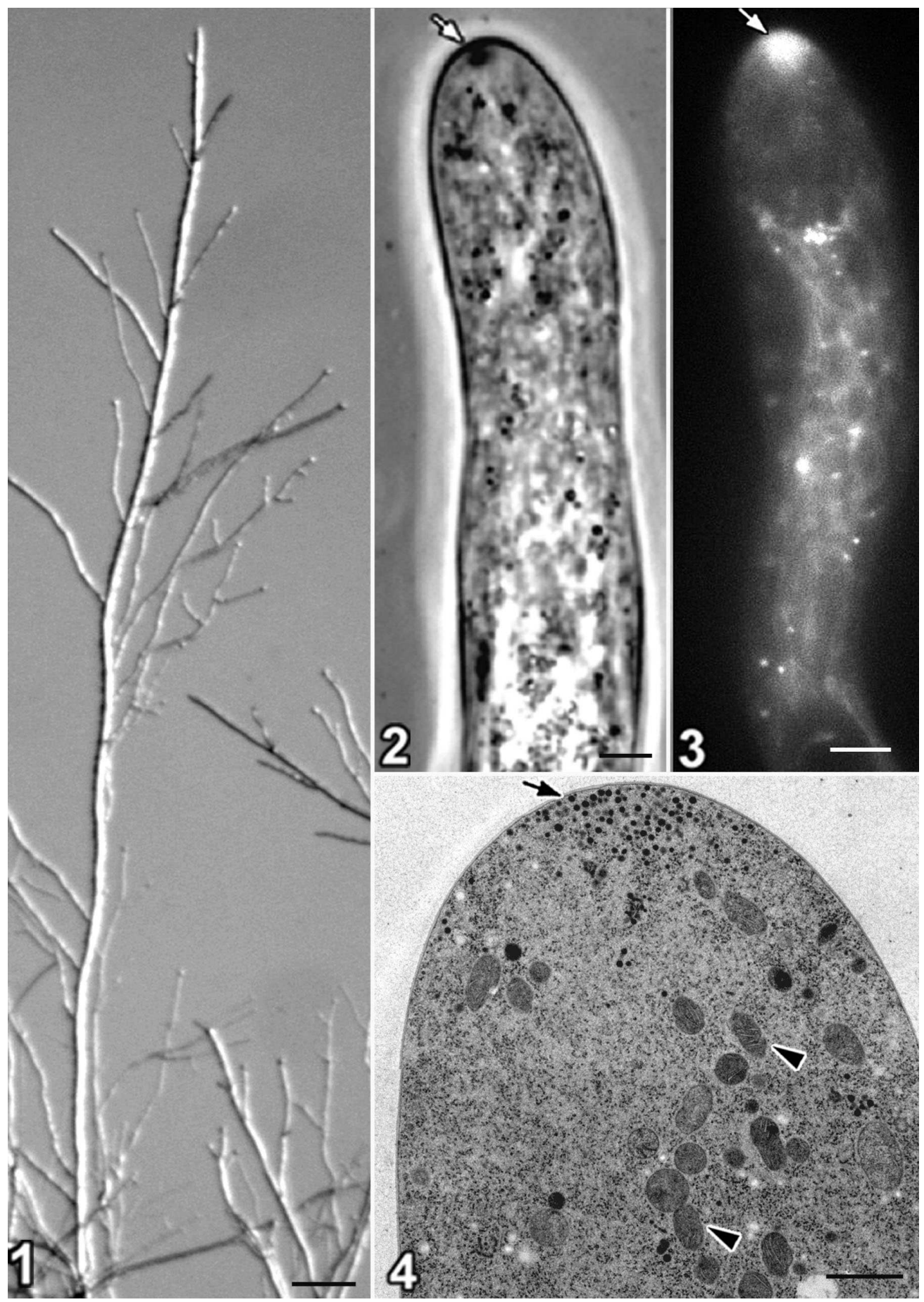

FIGURES 1-4. 1. Image of fungal hyphae growing on nutrient agar seen through dissecting microscope. Scale bar $=250 \mu \mathrm{m}$. 2. Phase-contrast light micrograph of Basidiobolus ranarum with Spitzenkörper (white arrow) shown. Scale bar $=3.0 \mu \mathrm{m}$. 3. Hypha of B. ranarum stained with membrane dye FM4-64. Spitzenkörper (white arrow) is shown. Scale bar $=3.0 \mu \mathrm{m}$. 4. Near-median TEM section through hyphal tip of $B$. ranarum illustrating structural components of the Spitzenkörper (arrow) and mitochondria (arrowheads) in surrounding cytoplasm. Scale bar $=1.5 \mu \mathrm{m}$. 